\title{
ON DECOMPOSABLE AND WARPED PRODUCT GENERALIZED QUASI EINSTEIN MANIFOLDS
}

\author{
Prajjwal Pal and Sahanous Mallick
}

\begin{abstract}
The object of the present paper is to study decomposable and warped product generalized quasi Einstein manifolds.

Keywords: Einstein manifold; Warped product; Ricci tensor; Generalized quasiEinstein manifolds
\end{abstract}

\section{Introduction}

A Riemannian manifold $\left(M^{n}, g\right), n=\operatorname{dim} M \geq 2$, is said to be an Einstein manifold if the following condition

$$
R_{i j}=\frac{r}{n} g_{i j}
$$

holds on $M$, where $R_{i j}$ and $r$ denote the Ricci tensor and the scalar curvature of $\left(M^{n}, g\right)$, respectively. According to Besse([2], p. 132), (1.1) is called the Einstein metric condition. Einstein manifolds play an important role in Riemannian Geometry as well as in general theory of relativity. Also, Einstein manifolds form a natural subclass of various classes of Riemannian manifolds by a curvature condition imposed on their Ricci tensor ([2], p. 432-433). For instance, every Einstein manifold belongs to the class of Riemannian manifolds $\left(M^{n}, g\right)$ realizing the following relation:

$$
R_{i j}=\lambda g_{i j}+\mu A_{i} A_{j},
$$

where $\lambda, \mu \in \mathbb{R}$ and $A_{i}$ is a non-zero covariant vector. Moreover, different structures on Einstein manifolds have been studied by several authors.

A non-flat Riemannian manifold $\left(M^{n}, g\right) \quad(n>2)$ is defined to be a quasiEinstein manifold if its Ricci tensor $R_{i j}$ of type $(0,2)$ is not identically zero and satisfies the condition (1.2).

It is to be noted that Chaki and Maity [5] also introduced the notion of quasiEinstein manifolds in a different way. They have taken $\lambda$ and $\mu$ as scalars and

Received April 06, 2016; accepted July 20, 2016

2010 Mathematics Subject Classification. 53C25 
the non-zero covariant vector $A_{i}$ as a unit covariant vector. Such an n-dimensional manifold is denoted by the symbol $(Q E)_{n}$. Quasi-Einstein manifolds have been studied by several authors such as De and Ghosh ([9], [10], [11], [12]), Ghosh, De and Binh [16], De and De [8], Debnath and Konar [14], Bejan and Binh [1] and many others.

Quasi-Einstein manifolds arose during the study of exact solutions of the Einstein field equations, as well as during considerations of quasi-umbilical hypersurfaces of semi-Euclidean manifolds. For instance, the Robertson-Walker space-time are quasi-Einstein manifolds. Also, quasi-Einstein manifold can be taken as a model of the perfect fluid space-time in general relativity [12]. So quasi-Einstein manifolds have some importance in the general theory of relativity.

Quasi-Einstein manifolds have been generalized by several authors in several ways such as generalized quasi-Einstein manifolds ([6], [10], [19]), super quasi Einstein manifolds ([7], [13], [21]), N(k)-quasi-Einstein manifolds ([17], [20], [25]) and many others. Also in [24] quasi-Einstein warped products have been studied by Sular and $\ddot{O}_{z g} \ddot{u r}$.

In a recent paper De and Ghosh [10] introduced the notion of generalized quasi Einstein manifolds. A non-flat Riemannian manifold is called a generalized quasi Einstein manifold if its Ricci tensor $R_{i j}$ of type $(0,2)$ is non-zero and satisfies the condition

$$
R_{i j}=\lambda g_{i j}+\mu A_{i} A_{j}+\nu B_{i} B_{j},
$$

where $\lambda, \mu$ and $\nu$ are certain non-zero scalars and $A_{i}, B_{i}$ are two orthogonal unit covariant vectors such that $g^{i j} A_{i} A_{j}=1, g^{i j} B_{i} B_{j}=1$ and $g^{i j} A_{i} B_{j}=0$. The vectors $A_{i}$ and $B_{i}$ are called the generators of the manifold and $\lambda, \mu$ and $\nu$ are called the associated scalars. Such a manifold is denoted by $G(Q E)_{n}$. If $\nu=0$, then the manifold reduces to a quasi Einstein manifold. $G(Q E)_{n}$ arose during the study of 2-quasi umbilical hypersurface of a Euclidean space [10]. In 2011, De and Mallick [15] prove the existence of $G(Q E)_{n}$ by several examples. Motivated by the above studies, the authors study the decomposability and warped product of $G(Q E)_{n}$.

The paper is organized as follows:

First, we state some examples of $G(Q E)_{n}$. Then in Section 3, we study a decomposable generalized quasi Einstein manifold. Section 4 deals with a $G(Q E)_{n}$ warped product manifold. Finally, we consider a $G(Q E)_{n}$ warped product manifold, base of which is unit dimensional.

\section{Examples of $G(Q E)_{n}$}

Example 2.1. [15] A 2-quasi-umbilical hypersurface of a space of constant curvature is a $G(Q E)_{n}$, which is not a quasi-Einstein manifold.

Example 2.2. [15] A quasi-umbilical hypersurface of a Sasakian space form is a $G(Q E)_{n}$, which is not a quasi-Einstein manifold. 
Example 2.3. De and Mallick [15] considered a Riemannian metric $g$ on $R^{4}$ by

$$
d s^{2}=g_{i j} d x^{i} d x^{j}=\left(x^{4}\right)^{\frac{4}{3}}\left[\left(d x^{1}\right)^{2}+\left(d x^{2}\right)^{2}+\left(d x^{3}\right)^{2}\right]+\left(d x^{4}\right)^{2} .
$$

Then they showed that $\left(M^{4}, g\right)$ is a generalized quasi-Einstein manifold, which is not a quasi-Einstein manifold.

Example 2.4. [22] $\ddot{O}_{z g} \ddot{u r}$ and Sular assumed an isometrically immersed surface $\bar{M}$ in $E^{3}$ with non-zero distinct principal curvatures $\lambda$ and $\mu$. Then they considered the hypersurface $M=\bar{M} \times E^{n-2}$ in $E^{n+1}, n \geq 4$. The principal curvatures of $M$ are $\tilde{\lambda}, \tilde{\mu}, 0, \ldots, 0$, where 0 occures (n-2)-times. Hence the manifold is a 2-quasi umbilical hypersurface and so it is generalized quasi-Einstein.

Example 2.5. [22] Özgür and Sular assumed a sphere $S^{2}$ in $E^{k+2}$ given by the immersion $f: S^{2} \rightarrow E^{k+2}$ and $B S^{2}$ be the bundle of unit normal to $S^{2}$. The hypersurface $M$ defined by the map $\varphi_{t}: B S^{2} \rightarrow E^{k+2}, \varphi_{t}(x, \xi)=F(x, t \xi)=f(x)+t \xi$ is called the tube of radius $t$ over $S^{2}$. It was proved in [4] that if $(\lambda, \lambda)$ are the principal curvature of $S^{2}$ then the principal curvatures of $M$ are $\left(\frac{\lambda}{1-t \lambda}, \frac{\lambda}{1-t \lambda},-\frac{1}{t}, \ldots,-\frac{1}{t}\right)$, where $-\frac{1}{t}$ occures $(k-1)$-times. So $M$ is 2-quasi umbilical and hence it is generalized quasi-Einstein.

\section{Decomposable $G(Q E)_{n}$}

A Riemannian manifold $\left(M^{n}, g\right)$ is said to be decomposable or a product manifold [23] if it can be expressed as $M_{1}^{p} \times M_{2}^{n-p}$ for $2 \leq p \leq(n-2)$, that is, in some coordinate neighbourhood of the Riemannian space $\left(M^{n}, g\right)$, the metric can be expressed as

$$
d s^{2}=g_{i j} d x^{i} d x^{j}=\bar{g}_{a b} d x^{a} d x^{b}+g_{\alpha \beta}^{*} d x^{\alpha} d x^{\beta},
$$

where $\bar{g}_{a b}$ are functions of $x^{1}, x^{2}, \ldots, x^{p}$ denoted by $\bar{x}$ and $g_{\alpha \beta}^{*}$ are functions of $x^{p+1}, x^{p+2}, \ldots, x^{n}$ denoted by $x^{*} ; a, b, c, \ldots$ run from 1 to $\mathrm{p}$ and $\alpha, \beta, \gamma, \ldots$ run from $\mathrm{p}+1$ to $\mathrm{n}$.

The two parts of (3.1) are the metrics of $M_{1}^{p}(p \geq 2)$ and $M_{2}^{n-p}(n-p \geq 2)$ which are called the components of the decomposable manifold $M^{n}=M_{1}^{p} \times M_{2}^{n-p}(2 \leq$ $p \leq n-2)$.

Let $\left(M^{n}, g\right)$ be a Riemannian manifold such that $M_{1}^{p}(p \geq 2)$ and $M_{2}^{n-p}(n-$ $p \geq 2$ ) are components of this manifold. Here throughout this section each object denoted by a 'bar' is assumed to be from $M_{1}$ and each object denoted by 'star' is assumed to be from $M_{2}$.

Then in a decomposable Riemannian manifold $M^{n}=M_{1}^{p} \times M_{2}^{n-p}(2 \leq p \leq n-2)$, the following relations hold [26]:

$R_{a b}=\bar{R}_{a b} ; R_{\alpha \beta}=R_{\alpha \beta}^{*} ; R_{a \alpha}=0 ; r=\bar{r}+r^{*}$,

where $r, \bar{r}$ and $r^{*}$ are scalar curvatures of $M, M_{1}$ and $M_{2}$ respectively.

Let us consider a Riemannian manifold $\left(M^{n}, g\right)$, which is a decomposable $G(Q E)_{n}$.

Then $M^{n}=M_{1}^{p} \times M_{2}^{n-p}(2 \leq p \leq n-2)$. Now from (1.3) we get

$$
\bar{R}_{a b}=\lambda \bar{g}_{a b}+\mu \bar{A}_{a} \bar{A}_{b}+\nu \bar{B}_{a} \bar{B}_{b}
$$


and

$$
R_{\alpha \beta}^{*}=\lambda g_{\alpha \beta}^{*}+\mu A_{\alpha}^{*} A_{\beta}^{*}+\nu B_{\alpha}^{*} B_{\beta}^{*},
$$

where

$$
A_{i}(x)=\left\{\begin{array}{cl}
\bar{A}_{i} & \text { for } \mathrm{i}=1,2, \ldots, \mathrm{p} \\
A_{i}^{*} & \text { for } \mathrm{i}=\mathrm{p}+1, \ldots, \mathrm{n}
\end{array}\right.
$$

Also we have

$$
R_{a \alpha}=\lambda g_{a \alpha}+\mu \bar{A}_{a} A_{\alpha}^{*}+\nu \bar{B}_{a} B_{\alpha}^{*} .
$$

which implies that

$$
\mu \bar{A}_{a} A_{\alpha}^{*}+\nu \bar{B}_{a} B_{\alpha}^{*}=0 .
$$

If possible, let

$$
\mu \bar{A}_{a} A_{\alpha}^{*}=0,
$$

which implies

$$
\bar{A}_{a} A_{\alpha}^{*}=0,
$$

since $\mu \neq 0$. Hence

$$
\text { either } \bar{A}_{a}=0 \text { or } A_{\alpha}^{*}=0
$$

(but not both, since $A_{i}$ is no more a unit vector).

Using (3.7) in (3.6) we get

$$
\nu \bar{B}_{a} B_{\alpha}^{*}=0
$$

which implies

$$
\bar{B}_{a} B_{\alpha}^{*}=0
$$

since $\nu \neq 0$. Therefore

$$
\text { either } \bar{B}_{a}=0 \text { or } B_{\alpha}^{*}=0,
$$

From (3.9) and (3.12) we have four cases as follows:

Case I: $\bar{A}_{a}=0$ and $\bar{B}_{a}=0$,

Case II: $A_{\alpha}^{*}=0$ and $B_{\alpha}^{*}=0$,

Case III: $\bar{A}_{a}=0$ and $B_{\alpha}^{*}=0$,

Case IV: $A_{\alpha}^{*}=0$ and $\bar{B}_{a}=0$.

Now if possible let $\bar{A}_{a}=0$ and $\bar{B}_{a}=0$, then (3.2) reduces to

$$
\bar{R}_{a b}=\lambda \bar{g}_{a b}
$$

This shows that the manifold $M_{1}^{p}$ is an Einstein manifold. On the other hand, if possible let $A_{\alpha}^{*}=0$ and $B_{\alpha}^{*}=0$, then (3.3) reduces to

$$
R_{\alpha \beta}^{*}=\lambda g_{\alpha \beta}^{*} \text {. }
$$

As above (3.14) shows that the manifold $M_{2}^{n-p}$ is an Einstein manifold.

Obviously the other cases are trivial. We get the similar results if we assume that (3.10) holds.

Thus we have the follwing: 
Theorem 3.1. If a $G(Q E)_{n}$ is a decomposable Riemannian manifold $\left(M^{n}, g\right)$ such that $M=M_{1}^{p} \times M_{2}^{n-p},(2 \leq p \leq n-2)$, and either (3.7) or (3.10) holds, then one component of the decomposable manifold is an Einstein manifold and the other is a generalized quasi Einstein manifold.

\section{4. $G(Q E)_{n}$ warped product manifolds}

The study of warped product manifold was initiated by Kručkovič [18] in 1957. Again in 1969 Bishop and O'Neill [3] also obtained the same notion of the warped product manifolds while they were constructing a large class of manifolds of negative curvature. Warped product are generalizations of the Cartesian product of Riemannian manifolds. Let $(\bar{M}, \bar{g})$ and $\left(M^{*}, g^{*}\right)$ be two Riemannian manifolds. Let $\bar{M}$ and $M^{*}$ be covered with coordinate charts $\left(U ; x^{1}, x^{2}, \ldots ., x^{p}\right)$ and $\left(V ; y^{p+1}, y^{p+2}, \ldots ., y^{n}\right)$ respectively.

Then the warped product $M=\bar{M} \times{ }_{f} M^{*}$ is the product manifold of dimension $n$ furnished with the metric

$$
g=\pi^{*}(\bar{g})+(f \circ \pi) \sigma^{*}\left(g^{*}\right)
$$

where $\pi: M \rightarrow \bar{M}$ and $\sigma: M \rightarrow M^{*}$ are natural projections such that the warped product manifold $\bar{M} \times{ }_{f} M^{*}$ is covered with the coordinate chart

$$
\left(U \times V ; x^{1}, x^{2}, \ldots, x^{p}, x^{p+1}=y^{p+1}, x^{p+2}=y^{p+2}, \ldots ., x^{n}=y^{n}\right) .
$$

Then the local components of the metric $g$ with respect to this coordinate chart are given by

$$
g_{i j}= \begin{cases}\bar{g}_{i j} & \text { for } \mathrm{i}=\mathrm{a} \text { and } \mathrm{j}=\mathrm{b} \\ f g_{i j}^{*} & \text { for } i=\alpha \text { and } j=\beta \\ 0 & \text { otherwise }\end{cases}
$$

Here $a, b, c, \ldots \in\{1,2, \ldots, p\}$ and $\alpha, \beta, \gamma, \ldots \in\{p+1, p+2, \ldots, n\}$ and $i, j, k, \ldots \in$ $\{1,2, \ldots, n\}$. Here $\bar{M}$ is called the base, $M^{*}$ is called the fiber and $f$ is called warping function of the warped product $M=\bar{M} \times_{f} M^{*}$. We denote by $\Gamma_{j k}^{i}, R_{i j k l}, R_{i j}$ and $r$ as the components of Levi-Civita connection $\nabla$, the Riemann-Christoffel curvature tensor $R$, Ricci tensor $S$ and the scalar curvature of $(M, g)$ respectively. Moreover we consider that, when $\Omega$ is a quantity formed with respect to $g$, we denote by $\bar{\Omega}$ and $\Omega^{*}$, the similar quantities formed with respect to $\bar{g}$ and $g^{*}$ respectively. Then the non-zero local components of Levi-Civita connection $\nabla$ of $(M, g)$ are given by

$$
\Gamma_{b c}^{a}=\bar{\Gamma}_{b c}^{a}, \quad \Gamma_{\beta \gamma}^{\alpha}=\Gamma_{\beta \gamma}^{* \alpha}, \quad \Gamma_{\beta \gamma}^{a}=-\frac{1}{2} \bar{g}^{a b} f_{b} g_{\beta \gamma}^{*}, \quad \Gamma_{a \beta}^{\alpha}=\frac{1}{2 f} f_{a} \delta_{\beta}^{\alpha},
$$

where $f_{a}=\partial_{a} f=\frac{\partial f}{\partial x^{a}}$. The local components $R_{h i j k}=g_{h l} R_{i j k}^{l}=g_{h l}\left(\partial_{k} \Gamma_{i j}^{l}-\right.$ $\left.\partial_{j} \Gamma_{i k}^{l}+\Gamma_{i j}^{m} \Gamma_{m k}^{l}-\Gamma_{i k}^{m} \Gamma_{m j}^{l}\right), \partial_{k}=\frac{\partial}{\partial x^{k}}$, of the Riemann-Christoffel curvature tensor $R$ of $(M, g)$ which may not vanish identically are the following:

$$
R_{a b c d}=\bar{R}_{a b c d}, R_{a \alpha b \beta}=-f T_{a b} g_{\alpha \beta}^{*}, R_{\alpha \beta \gamma \delta}=f R_{\alpha \beta \gamma \delta}^{*}-f^{2} P G_{\alpha \beta \gamma \delta}^{*},
$$


where $G_{i j k l}=g_{i l} g_{j k}-g_{i k} g_{j l}$ and

$T_{a b}=-\frac{1}{2 f}\left(\nabla_{b} f_{a}-\frac{1}{2 f} f_{a} f_{b}\right), \quad \operatorname{tr}(T)=g^{a b} T_{a b}, P=\frac{1}{4 f^{2}} g^{a b} f_{a} f_{b}$. Again the nonzero local components of the Ricci tensor $R_{j k}=g^{i l} R_{i j k l}$ of $(M, g)$ are given by

$$
R_{a b}=\bar{R}_{a b}+(n-p) T_{a b}, \quad R_{\alpha \beta}=R_{\alpha \beta}^{*}-Q g_{\alpha \beta}^{*},
$$

where $Q=f((n-p-1) P-\operatorname{tr}(T))$. The scalar curvature $r$ of $(M, g)$ is given by

$$
r=\bar{r}+\frac{r^{*}}{f}-(n-p)[(n-p-1) P-2 \operatorname{tr}(T)] .
$$

Let $M=\bar{M} \times_{f} M^{*}$ be a non-flat warped product manifold and also let $M$ be a $G(Q E)_{n}$. That is,

$$
\text { (4.7) } \quad R_{a b}=\lambda g_{a b}+\mu A_{a} A_{b}+\nu B_{a} B_{b} \text {. }
$$

From (4.7), using (4.5) we get

$$
\bar{R}_{a b}+(n-p) T_{a b}=\lambda \bar{g}_{a b}+\mu \bar{A}_{a} \bar{A}_{b}+\nu \bar{B}_{a} \bar{B}_{b},
$$

where

$$
A_{i}(x)= \begin{cases}\bar{A}_{i} & \text { for } \mathrm{i}=1, \ldots, \mathrm{p} \\ A_{i}^{*} & \text { otherwise }\end{cases}
$$

and

$$
B_{i}(x)= \begin{cases}\bar{B}_{i} & \text { for } \mathrm{i}=1, \ldots, \mathrm{p} \\ B_{i}^{*} & \text { otherwise }\end{cases}
$$

Then from (4.8) we get

$$
\bar{R}_{a b}=\lambda \bar{g}_{a b}+\mu \bar{A}_{a} \bar{A}_{b}+\nu \bar{B}_{a} \bar{B}_{b}-(n-p) T_{a b},
$$

If possible, we assume that $\bar{M}$ is also $G(Q E)_{p}$, then from (4.11) we get

$$
T_{a b}=0 .
$$

Conversely, if (4.12) holds, then from (4.11) we can conclude that $\bar{M}$ is a $G(Q E)_{p}$. Thus we have the following:

Theorem 4.1. $\quad M=\bar{M} \times{ }_{f} M^{*}$ is a $G(Q E)_{n}$ warped product manifold, if and only if $\bar{M}$ is a $G(Q E)_{p}$ provided $T_{a b}=0$.

Now if in particular

$$
T_{a b}=k \bar{g}_{a b},
$$

where $k \neq 0$ is some constant. Then (4.11) takes the form

$$
\bar{R}_{a b}=\{\lambda-k(n-p)\} \bar{g}_{a b}+\mu \bar{A}_{a} \bar{A}_{b}+\nu \bar{B}_{a} \bar{B}_{b},
$$

where $A_{i}$ and $B_{i}$ are defined by (4.9) and (4.10) from which it follwos that $\bar{M}$ is a $G(Q E)_{p}$. Conversely, if $\bar{M}$ is a $G(Q E)_{n}$ then using (4.14) in (4.11) we get (4.13). Thus we have the following: 
Theorem 4.2. $M=\bar{M} \times_{f} M^{*}$ is a $G(Q E)_{n}$ warped product manifold, then $\bar{M}$ is $a G(Q E)_{p}$ if and only if (4.13) holds.

Similarly, we get from (4.7)

$$
R_{\alpha \beta}=\lambda g_{\alpha \beta}+\mu A_{\alpha} A_{\beta}+\nu B_{\alpha} B_{\beta} .
$$

Using (4.5), (4.15) yields

$$
R_{\alpha \beta}^{*}=(\lambda f+Q) g_{\alpha \beta}^{*}+\mu A_{\alpha}^{*} A_{\beta}^{*}+\nu B_{\alpha}^{*} B_{\beta}^{*} .
$$

Hence $M^{*}$ is a $G(Q E)_{n-p}$.

Converse is trivial. Thus we have the following:

Theorem 4.3. $\quad M=\bar{M} \times_{f} M^{*}$ is a $G(Q E)_{n}$ warped product manifold, if and only if $M^{*}$ is a generalized quasi-Einstein manifold of dimension (n-p).

\section{5. $G(Q E)_{n}$ warped product manifolds with unit dimensional base}

In this section, we consider $G(Q E)_{n}$ warped product manifolds $M=I \times{ }_{f} M^{*}$, $\operatorname{dim} I=1, \operatorname{dim} M^{*}=n-1(n \geq 3), f=\exp \left\{\frac{q}{2}\right\}$. We take the metric on $I$ as $(d t)^{2}$. Using the above consideration and (4.5), we get

$$
R_{t t}=\bar{R}_{t t}-\frac{(n-1)}{16}\left[4 q^{\prime \prime}+\left(q^{\prime}\right)^{2}\right]
$$

which implies

$$
R_{t t}=-\frac{(n-1)}{16}\left[4 q^{\prime \prime}+\left(q^{\prime}\right)^{2}\right]
$$

since $\bar{R}_{t t}$ of $I$ is zero. Also

$$
R_{\alpha \beta}=R_{\alpha \beta}^{*}-\frac{e^{\frac{q}{2}}}{16}\left[4(n-1) q^{\prime \prime}+(2 n-3)\left(q^{\prime}\right)^{2}\right] g_{\alpha \beta}^{*},
$$

where " and "I" denote the 1st order and 2nd order partial derivative respectively, with respect to 't'. Since $M$ is a generalized quasi Einstein manifold, from (1.3) we have

$$
R_{t t}=\lambda g_{t t}+\mu A_{t} A_{t}+\nu B_{t} B_{t}
$$

and

$$
R_{\alpha \beta}=\lambda g_{\alpha \beta}+\mu A_{\alpha} A_{\beta}+\nu B_{\alpha} B_{\beta},
$$

where we take $A_{i}$ and $B_{i}$ as defined in (4.9) and (4.10). Now since $\operatorname{dim} I=1$, we can take

$$
\bar{A}_{t}=l \text { and } \bar{B}_{t}=m,
$$

where $l$ and $m$ are functions on $M$. Using (4.1), (4.2), (4.9), (4.10) and (5.6), the equations (5.4) and (5.5) reduce to

$$
R_{t t}=\lambda+\mu l^{2}+\nu m^{2}
$$


and

$$
R_{\alpha \beta}=\lambda e^{\frac{q}{2}} g_{\alpha \beta}^{*}+\mu A_{\alpha}^{*} A_{\beta}^{*}+\nu B_{\alpha}^{*} B_{\beta}^{*} .
$$

From (5.2) and (5.7) we get

$$
\lambda+\mu l^{2}+\nu m^{2}=-\frac{(n-1)}{16}\left[4 q^{\prime \prime}+\left(q^{\prime}\right)^{2}\right] .
$$

Again from (5.3) and (5.8) we obtain

$$
R_{\alpha \beta}^{*}=\frac{e^{\frac{q}{2}}}{16}\left[4(n-1) q^{\prime \prime}+(2 n-3)\left(q^{\prime}\right)^{2}+16 \lambda\right] g_{\alpha \beta}^{*}+\mu A_{\alpha}^{*} A_{\beta}^{*}+\nu B_{\alpha}^{*} B_{\beta}^{*},
$$

where $\lambda, \mu$ and $\nu$ are related by (5.9). Thus (5.10) implies that $M^{*}$ is a generalized quasi Einstein manifold. Hence we have the following:

Theorem 5.1. If $M=I \times_{f} M^{*}$, is a $G(Q E)_{n}$ warped product manifold and $\operatorname{dim} I=1, \operatorname{dim} M^{*}=n-1(n \geq 3)$, then $M^{*}$ is a generalized quasi Einstein manifold.

Now, we consider warped product manifolds $M=I \times{ }_{f} M^{*}, \operatorname{dim} I=1, \operatorname{dim} M^{*}=$ $n-1(n \geq 3), f=\exp \left\{\frac{q}{2}\right\}$ and $M^{*}$ is a $(Q E)_{n}$. We take the metric on $I$ as $(d t)^{2}$. In this case, (5.2) and (5.3) can also be obtained using the above consideration and (4.5). Since $M^{*}$ is $(Q E)_{n}$, from $(1.2)$ we have

$$
R_{\alpha \beta}^{*}=\lambda g_{\alpha \beta}^{*}+\mu A_{\alpha}^{*} A_{\beta}^{*},
$$

where $\lambda$ and $\mu$ are certain non-zero scalars and $A_{i}^{*}$ is an unit covariant vector such that $g_{i j}^{*} A_{i}^{*} A_{j}^{*}=1$ and

$$
A_{i}(x)= \begin{cases}\bar{A}_{i} & \text { for } \mathrm{i}=1 \\ A_{i}^{*} & \text { otherwise }\end{cases}
$$

Using (5.11) in (5.3) we get

$$
R_{\alpha \beta}=\lambda g_{\alpha \beta}^{*}+\mu A_{\alpha}^{*} A_{\beta}^{*}-\frac{e^{\frac{q}{2}}}{16}\left[4(n-1) q^{\prime \prime}+(2 n-3)\left(q^{\prime}\right)^{2}\right] g_{\alpha \beta}^{*} .
$$

which implies

$$
R_{\alpha \beta}=-\frac{e^{\frac{q}{2}}}{16}\left[4(n-1) q^{\prime \prime}+(2 n-3)\left(q^{\prime}\right)^{2}\right] g_{\alpha \beta}^{*}+\lambda g_{\alpha \beta}^{*}+\mu A_{\alpha}^{*} A_{\beta}^{*} .
$$

Now using (4.2) and (5.12) in (5.14) we obtain

$$
R_{\alpha \beta}=-\frac{1}{16}\left[4(n-1) q^{\prime \prime}+(2 n-3)\left(q^{\prime}\right)^{2}\right] g_{\alpha \beta}+\frac{\lambda}{e^{\frac{q}{2}}} g_{\alpha \beta}+\mu A_{\alpha} A_{\beta} .
$$

Now if we choose $g_{\alpha \beta}=e^{\frac{q}{2}} B_{\alpha} B_{\beta}$, where

$$
B_{i}(x)= \begin{cases}\bar{B}_{i} & \text { for } \mathrm{i}=1 \\ B_{i}^{*} & \text { otherwise }\end{cases}
$$


Then (5.15) yields

$$
R_{\alpha \beta}=-\frac{1}{16}\left[4(n-1) q^{\prime \prime}+(2 n-3)\left(q^{\prime}\right)^{2}\right] g_{\alpha \beta}+\mu A_{\alpha} A_{\beta}+\lambda B_{\alpha} B_{\beta} .
$$

Again from (5.2) we get

$$
\begin{array}{r}
R_{t t}=\frac{1}{16}\left[4(n-1) q^{\prime \prime}+(2 n-3)\left(q^{\prime}\right)^{2}\right] g_{t t}-\frac{1}{16}\left[4(n-1) q^{\prime \prime}\right. \\
\left.+(2 n-3)\left(q^{\prime}\right)^{2}\right]-\frac{(n-1)}{16}\left[\left(q^{\prime}\right)^{2}+4 q^{\prime \prime}\right],
\end{array}
$$

since $\bar{g}_{t t}=1$ and $g_{t t}=\bar{g}_{t t}$ in $I$. Thus (5.18) can be written as

$$
R_{t t}=\frac{1}{16}\left[4(n-1) q^{\prime \prime}+(2 n-3)\left(q^{\prime}\right)^{2}\right] g_{t t}-\frac{(3 n-4)}{16}\left(q^{\prime}\right)^{2}+\frac{2(n-1)}{4} q^{\prime \prime}
$$

Since $\operatorname{dim} I=1$, we can take

$$
\bar{A}_{t}=q^{\prime} \text { and } \bar{B}_{t}=\sqrt{ } q^{\prime \prime},
$$

where $q^{\prime}$ and $q^{\prime \prime}$ are functions on $M$. Then using (5.12),(5.16) and (5.20) we can write (5.19) as follows:

$$
\left(5.21 R_{t t}=\frac{1}{16}\left[4(n-1) q^{\prime \prime}+(2 n-3)\left(q^{\prime}\right)^{2}\right] g_{t t}-\frac{(3 n-4)}{16} A_{t} A_{t}+\frac{2(n-1)}{4} B_{t} B_{t} .\right.
$$

Thus from (5.17) and (5.21) we caconclude that $M=I \times_{f} M^{*}$ is a generalized quasi Einstein manifold if $M^{*}$ is a quasi Einstein manifold. Hence we have the following:

Theorem 5.2. If $M=I \times_{f} M^{*}$, is a warped product manifold and $\operatorname{dim} I=1$, $\operatorname{dim} M^{*}=n-1(n \geq 3)$ and $M^{*}$ is a quasi Einstein manifold, then $M$ is a generalized quasi Einstein manifold.

\section{REF E R E N C E S}

1. C. L. BeJAN and T. Q. BINH:Generalized Einstein manifolds, WSPCProceedings, Trim Size, dga 2007, 47 - 54 .

2. A. L. Besse: Einstein manifolds, Ergeb. Math. Grenzgeb., Folge, Bd. 10, Springer-Verlag, Berlin, Heidelberg, New York, 1987.

3. R. L. Bishop and B. O'Neill: Manifolds of negative curvature, Trans. Amer. Math. Soc., 145(1969), $1-49$.

4. T. E. CECIL and P. J. RYAN: Tight and Taut Immersions of Manifolds, Research Notes in Mathematics, 107, Pitman (Advanced Publishing Program), Boston, M. A., 1985.

5. M. C. Chaki and R. K. Maity: On quasi Einstein manifolds, Publ. Math. Debrecen, 57(2000), $297-306$. 
6. M. C. Chaki: On generalized quasi Einstein manifolds, Publ. Math. Debrecen, $\mathbf{5 8}(2001), 683-691$.

7. M. C. ChaкI: On super quasi-Einstein manifolds, Publ. Math. Debrecen, 64(2004), $481-488$.

8. U. C. De and B. K. DE: On quasi-Einstein manifolds, Commun. Korean Math. Soc., 23(2008), $413-420$.

9. U. C. De and G. C. Ghosh: On quasi Einstein manifolds, Periodica Math. Hungarica, 48(2004), $223-231$.

10. U. C. De and G. C. Ghosh: On generalized quasi Einstein manifolds, Kyungpook Math. J., 44(2004), $607-615$.

11. U. C. De and G. C. Ghosh: On conformally flat special quasi Einstein manifolds, Publ. Math. Debracen, 66(2005), 129 - 136.

12. U. C. De and G. C. Ghosh: On quasi-Einstein and special quasi-Einstein manifolds, Proc. of the Int. Conf. of Mathematics and its applications, Kuwait University, April 5 - 7, 2004, 178 - 191.

13. P. Debnath and A. Konar: On super quasi-Einstein manifolds, Publications de L'institute Mathematique, Nouvelle serie, Tome 89(2011), 95 - 104.

14. P. Debnath and A. Konar: On quasi-Einstein manifolds and quasi-Einstein spacetimes, Differ. Geom. Dyn. Syst., 12(2010), $73-82$.

15. U. C. DE and S. MALLICK: On the existence of generalized quasi Einstein manifolds, Archivum Mathematicum (Brno), Tomus, 47(2011), 279 - 291.

16. G. C. Ghosh, U. C. De and T. Q. Binh: Certain curvature restrictions on a quasi-Einstein manifold, Publ. Math. Debrecen, 69(2006), 209 - 217.

17. A. A. Hosseinzadeh and A. TAleshian: On conformal and quasi-conformal curvature tensors of an $N(k)$-quasi-Einstein manifold, Commun. Korean Math. Soc., 27(2012), $317-326$.

18. G. I. KRuČKovič: On semi-reducible Riemannian spaces, Dokl. Akad. Nauk SSSR 115(1957), 862 - 865 (in Russian).

19. C. ÖzGüR: On a class of generalized quasi-Einstein manifolds, Applied Sciences, Balkan Society of Geometers, Geometry Balkan Press, 8(2006), 138 - 141.

20. C. ÖzGüR: $N(k)$-quasi-Einstein manifolds satisfying certain conditions, Chaos, Solitons and Fractals, 38(2008), 1373 - 1377.

21. C. ÖzGür: On some classes of super quasi-Einstein manifolds, Chaos, Solitons and Fractals, 40(2009), 1156 - 1161.

22. C. ÖZGür and S. SUlAR: On some properties of generalized quasi-Einstein manifolds, Indian Journal of Mathematics, 50(2008), 297 - 302.

23. J. A. Schouten: Ricci-Calculus, An introduction to Tensor Analysis and its Geometrical Applications, Springer-Verlag, Berlin-Göttingen-Heidelberg, 1954.

24. S. Sular and C. ÖzG'Ur: On quasi-Einstein warped products, Ann. St. Univ. "Al I Cuza" Iasi (S.N.), Tomul 58(2012), 353 - 362.

25. A. Taleshian and A. A. Hosseinzadeh: Investigation of Some Conditions on $N(k)$-quasi-Einstein manifolds, Bull. Malays. Math. Soc., 34(2011), $455-464$.

26. K. YANO and M. Kon: Structures on manifolds, World scientific, Singapore, $1984,418-421$. 
Prajjwal Pal

Chakdaha Co-operative Colony Vidyayatan(H.S)

P.O.-Chakdaha, Dist-Nadia

West Bengal, PIN-741222, India

prajjwalpal@yahoo.in

Sahanous Mallick

Department of Mathematics

Chakdaha College

P.O.-Chakdaha, Dist-Nadia

West Bengal, PIN-741222, India

sahanousmallick@gmail.com 